\section{General summary}

\author{
A Martin and $\mathrm{J}$ Licinio \\ UCLA, Los Angeles, CA, USA
}

\section{NEWS \& COMMENTARY}

The role of genes and family in trauma exposure and posttraumatic stress disorder

$S$ Seedat, DJ Niehaus, DJ Stein

The importance of heredity is well established for a variety of psychiatric disorders, including schizophrenia, bipolar disorder, and alcoholism, where genetic linkage has been shown and replicated in studies. The authors briefly review here the limited data on genetic and family influences in posttraumatic stress disorder.

\section{MILLENNIUM ARTICLE}

Putative endophenotypes of dysthymia: evolutionary, clinical and pharmacogenomic considerations AB Niculescu, HS Akiskal

Dysthymia is characterized by helplessness, low drive, and decreased joie de vivre. In evolutionary terms, it may confer some advantage in dealing with ongoing unresolvable stress and failure. In excess, it is maladaptive: dysthymia requiring clinical intervention, involves $3-5 \%$ of the population, and is more common in women. Once considered a character flaw, it has emerged as a subthreshold trait of biological importance in predisposing to major mood disorders. This review integrates two decades of research in understanding the nature of this trait affective condition.

\section{IMMEDIATE COMMUNICATIONS}

\section{MAO-A and COMT polymorphisms and gene effects in narcolepsy \\ Y Dauvilliers, E Neidhart, M Lecendreux, M Billiard, M Tafti}

The authors investigated polymorphisms of MAO-A and COMT in 97 narcoleptic patients and sought for association with disease symptoms. No evidence of association between genotype or allele frequencies of the two genes and narcolepsy was found. However women narcoleptics with high COMT activity fell asleep twice faster than those with low COMT activity during the multiple sleep latency test (MSLT) while the opposite was true for men. COMT genotype also strongly affected the presence of sleep paralysis and the number of REM sleep onsets during the MSLT. These findings constitute the first genetic evidence for the critical involvement of the dopaminergic and/or noradrenergic systems in human narcolepsy.

\author{
RNA editing of the $5-\mathrm{HT}_{2 \mathrm{C}}$ receptor is reduced in \\ schizophrenia \\ MS Sodhi, PWJ Burnet, AJ Makoff, RW Kerwin, \\ PJ Harrison
}

RNA editing is an enzymic process which modifies RNA sequence, often producing amino acid substitutions and altered protein function. This phenomenon occurs in the messenger RNA (mRNA) of the $5-\mathrm{HT}_{2 \mathrm{C}}$ receptor $\left(5-\mathrm{HT}_{2 \mathrm{C}} \mathrm{R}\right)$, leading to pharmacologically different $5-\mathrm{HT}_{2 \mathrm{C}} \mathrm{R}$ variants. In the current study, analysis of mRNA transcripts from the frontal cortex detected an overall reduction of $5-\mathrm{HT}_{2 \mathrm{C}} \mathrm{R}$ RNA editing in schizophrenia subjects compared with controls. Interestingly, the unedited isoform was significantly increased in schizophrenia. These results illustrate that potentially important receptor alterations may occur in psychiatric illness which are not detectable in terms of genetic association or receptor abundance.

\section{ORIGINAL RESEARCH ARTICLES}

The expression of glutamate transporter GLT-1 in the rat cerebral cortex is downregulated by the antipsychotic drug clozapine

$M$ Melone, L Vitellaro-Zuccarello, A Vallejo-

Illarramendi, A Pérez-Samartin, C Matute, A Cozzi, DE Pellegrini-Giampietro, JD Rothstein, F Conti

The authors report that the antipsychotic clozapine reduced immunoreactivity to the glutamate transporter GLT-1 in several brain regions, that the effects were most evident after 9 weeks of treatment and that they were most evident in the frontal cortex, where the expression of GLT-1 protein is severely reduced. The authors also showed that both glutamate uptake and uptake-associated currents in Xenopus oocytes injected with mRNA extracted from the cerebral cortex of rats treated with clozapine were remarkably reduced in comparison to control. Finally, they showed that clozapine increased extracellular levels of glutamate in the frontal cortex. These findings indicate that clozapine potentiates glutamatergic synaptic transmission by regulating glutamate transport. Moreover, given that several studies suggest that glutamatergic hypofunction explains some features of schizophrenia, the present data suggest the possibility that changes in GLT-1 expression and/or activity may play a role in the pathophysiology of schizophrenia. 
354

Mutation analysis of SYNJ1: a possible candidate gene for chromosome 21q22-linked bipolar disorder T Saito, F Guan, DF Papolos, $S$ Lau, M Klein, CSJ Fann, HM Lachman

A number of genetic studies suggest that chromosome 21 , specifically, the region designated $21 \mathrm{q} 22$, has a gene that increases the susceptibility to develop bipolar disorder (BPD). One candidate gene that maps to chromosome 21q22, SYNJ1, codes for synaptojanin, a protein found in presynaptic terminals (the distal end of the nerve). The authors probed the SYNJ1 gene for mutations and analyzed their frequency in nearly 150 bipolar patients and a similar number of controls. Eleven mutations were found. Of these, several were found to be increased in patients with BPD, although the results fell short of statistical significance. The data suggest that SYNJ1 should be considered as a valid candidate gene for BPD but a larger sample size will have to be analyzed.

A genome screen of a large bipolar affective disorder pedigree supports evidence for a susceptibility locus on chromosome 13q

RF Badenhop, MJ Moses, A Scimone, PB Mitchell, KR Ewen, A Rosso, JA Donald, LJ Adams, PR Schofield

The authors undertook a $10 \mathrm{cM}$ genome screen in a large multigenerational bipolar pedigree consisting of 40 individuals, including six affecteds. They found strongest evidence for linkage to chromosome 13q14 with a maximum NPL score of $4.09(P=0.008)$ between markers D13S1272 and D13S153, a maximum twopoint LOD score of $2.91(\theta=0.0)$ for D13S153 and a maximum three-point LOD score of 3.0 between markers D13S291 and D13S153. Haplotype analysis enabled them to narrow the likely disease region to a $6 \mathrm{cM}$ region between markers D13S1272 and D13S1319, which contains the serotonin $2 \mathrm{~A}$ receptor candidate gene. Two single nucleotide polymorphisms were identified in this gene but the authors did not detect any significant differences in allele frequency in a casecontrol sample. The region on chromosome 13q14-32 has previously been implicated in other bipolar and schizophrenia cohorts. The authors' results provide further support for the existence of a susceptibility locus on chromosome 13q14.

\section{Common effects of chronically administered antipanic drugs on brainstem $\mathrm{GABA}_{\mathrm{A}}$ receptor subunit gene expression \\ VA-MI Tanay, AJ Greenshaw, GB Baker, AN Bateson}

Anti-panic therapeutics fall into various classes that exhibit markedly different primary sites of action but the mechanisms by which the therapeutic treatment of panic disorder is achieved are unknown. The GABA receptor is a known drug target for acute anxiety relief. Therefore whether the modulation of $\mathrm{GABA}_{\mathrm{A}}$ receptor gene expression is a common effect of long-term exposure to anti-panic agents was examined. Examples of different drug classes were used: alprazolam (benzodiazepine), phenelzine (monoamine oxidase inhibitor) and imipramine (tricyclic). Changes in the expression of specific $\mathrm{GABA}_{\mathrm{A}}$ receptor subunit genes were observed in rat brainstem that were common to all three anti-panic agents but not to buspirone, an anxiolytic with no anti-panic efficacy. The authors conclude that long-term treatment with anti-panic agents produces a remodeling of the $\mathrm{GABA}_{\mathrm{A}}$ receptor population and this may be involved in the mechanism of anti-panic therapeutic action.

\section{D10S1423 identifies a susceptibility locus for Alzheimer's disease in a prospective, longitudinal, double-blind study of asymptomatic individuals GS Zubenko, HB Hughes III, JS Stiffler}

This report describes the prospective, longitudinal, double-blind assessment of the age-specific risk of $\mathrm{AD}$ encountered by 325 asymptomatic first-degree relatives of AD probands who carried the D10S1423 234-bp allele, the APOE E4 allele, or both, after 11.5 years of systematic follow-up. The age-specific risk of developing $\mathrm{AD}$ was the greatest for individuals who carried both alleles. Cox proportional hazards models were developed to estimate the risk ratios for each genotype, controlling for the potential effects of age at recruitment, sex, and years of education. In the resulting best fitting model, only individuals who carried both risk alleles exhibited a risk ratio that differed significantly from 1 . After controlling for these genotypes, female gender was also significantly associated with increased risk of developing AD. Neither age at recruitment nor years of education made significant contributions to the model.

\section{Dopa decarboxylase gene polymorphisms and attention deficit hyperactivity disorder (ADHD): no evidence for association in the Irish population $Z$ Hawi, D Foley, A Kirley, M McCarron, $M$ Fitzgerald, M Gill}

Dopa decarboxylase (DDC) is an enzyme which catalyses the decarboxylation of dopa to dopamine and L5 hydroxytryptophan to serotonin. It has been suggested that genes involved in the dopaminergic system play a primary role in predisposing to attention deficit hyperactivity disorder (ADHD). In this study, the 4-bp insertion/deletion variant mapped to the first neuronally expressed exon 1 at the dopa decarboxylase gene and two microsatellite markers flanking the gene were investigated for possible association with ADHD. The authors observed an increased transmission (though not significant) of the 4-bp insertion (allele 1) to ADHD cases $\left(\chi^{2}=2.72, P=0.1\right.$, $\left.\mathrm{RR}=1.25\right)$. However a weak but marginally significant association between allele 10 (213 bp) of the $3^{\prime}$ microsatellite D7S2422 ( $0.75 \mathrm{cM}$ distal to dopa decarboxylase gene) and ADHD was observed $\left(\chi^{2}=4.2, P=0.04, \mathrm{RR}=1.48\right)$. This provides 
preliminary evidence that this locus or a closely mapped DNA variant may be involved in the genetic susceptibility to ADHD.

\section{Association of a dopamine transporter polymorphism and attention deficit hyperactivity disorder in UK and Turkish samples $S$ Curran, J Mill, E Tahir, L Kent, S Richards, A Gould, L Huckett, J Sharp, C Batten, S Fernando, $F$ Ozbay, Y Yazgan E Simonoff, $M$ Thompson, E Taylor, $P$ Asherson}

Molecular genetic studies in attention deficit hyperactivity disorder (ADHD) have focused on candidate genes within the dopamine system, which is thought to be the main site of action of stimulant drugs that are the primary pharmacological treatment of the disorder. Of particular interest are findings with the dopamine transporter gene (DAT1), since stimulant drugs interact directly with the transporter protein. The authors have analyzed the same VNTR marker in a dataset of UK Caucasian children and an independent dataset of Turkish Caucasian children with DSM-IV ADHD, using the transmission disequilibrium test (TDT). Results from the UK, but not the Turkish sample support association and linkage between genetic variation and the DAT1 locus and ADHD. When considered alongside evidence from other published reports, there is only modest evidence for the association, consistent with a very small main effect for the 480-bp allele; however, the authors find significant evidence of heterogeneity between the combined dataset.

Two dopamine genes related to reports of childhood retrospective inattention and conduct disorder

$D C$ Rowe, C Stever, D Chase, S Sherman,

A Abramowtiz, ID Waldman

Attention deficit disorder and conduct disorder are relatively prevalent psychiatric disorders in childhood. These psychiatric disorders are familial, with parents of affected children often reporting similar symptoms from their own childhoods. In this study, parents of clinic referred and control children reported retrospectively on inattention and conduct disorder symptoms in adolescence. The parents were also scored for variants of two dopamine-related genes that have been associated with attention deficit hyperactivity disorder in childhood. The authors report an association of the 7-repeat allele of the dopamine receptor D4 gene with fathers' inattention and conduct disorder symptoms. They also found an association of the 10-repeat genotype and mothers' inattention symptoms. These findings suggest that genetic associations found in children may be replicated in their parents. They also offer support to theories implicating dopaminergic-reward pathways in the brain in the etiology of externalizing psychiatric disorders.
Role of the serotonin transporter gene in the

behavioral expression of autism

$S$ Tordjman, L Gutknecht, M Carlier, E Spitz,

C Antoine, F Slama, V Carsalade, DJ Cohen,

P Ferrari, PL Roubertoux, GM Anderson

There has been a longstanding interest in the possible role of serotonin in autism. The authors have examined parent-offspring transmission of the long $(I)$ and short $(s)$ forms of a deletion/insertion variation in the serotonin transporter gene. In families of 71 children with autism, transmission of the variants did not differ between children with autism and their unaffected siblings. However, genetic transmission was dependent upon severity of impairments in the social and communication domains, with greater $s$ transmission in severely impaired individuals and greater $l$ transmission in mild/moderately impaired individuals. Thus, while the $s$ and $l$ forms of the gene do not appear to influence risk for autism, they may modify the severity of autistic impairment in social relatedness and communication. The results suggest that future research on the genetics of autism should carefully assess each of the major behavioral domains and seriously consider the possible role of modifying genes.

\section{Attention deficit hyperactivity (ADHD) and the dopamine $\mathrm{D} 4$ receptor gene: evidence for association but no linkage in a UK sample \\ J Mill, S Curran, L Kent, S Richards, A Gould, $V$ Virdee, L Huckett, J Sharp, C Batten, S Fernando, E Simonoff, M Thompson, J Zhao, P Sham, E Taylor, $P$ Asherson}

Recent studies report genetic association between attention deficit hyperactivity disorder (ADHD) and a repeat length variation of the dopamine D4 receptor gene (DRD4). The authors have attempted to replicate these findings in their sample of children with a diagnosis of ADHD from the UK. They found evidence for this association among 132 ADHD probands when compared with 189 controls. However, within-family tests of association which are robust to population stratification failed to support this finding. The authors therefore concluded that, at least in this sample, the case-control findings are likely to be falsely positive. However they cannot rule out alternative explanations of low statistical power and gene-environment correlation.

\section{Increased S100B blood levels in unmedicated and treated schizophrenic patients are correlated with negative symptomatology \\ $M$ Rothermundt, U Missler, $V$ Arolt, $M$ Peters, $J$ Leadbeater, $M$ Wiesmann, S Rudolf, KP Wandinger, H Kirchner}

$\mathrm{S} 100 \mathrm{~B}$, a protein produced by astroglial cells, is a marker of astroglial cellular integrity. It has been shown to be increased in acute brain damage and neurodegeneration. This study included 26 drug-free 
356

patients with acute schizophrenia and 26 matched healthy controls. S100B blood concentrations were determined upon admission and after 6 weeks of neuroleptic treatment. Unmedicated schizophrenic patients showed significantly increased S100B levels compared to matched healthy controls. After 6 weeks of treatment, 11 patients showed normal S100B levels while in 15 patients the levels remained increased. Those patients had significantly higher PANSS negative scores upon admission and after 6 weeks of treatment. These results show that continuously increased S100B levels are associated with negative symptomatology.

Further evidence for a bipolar risk gene on chromosome 12q24 suggested by investigation of haplotype sharing and allelic association in patients from the Faroe Islands

$B$ Degn, $M D$ Lundorf, $A$ Wang, $M$ Vang, $O$ Mors, TA Kruse, H Ewald

Linkage studies have suggested that a risk gene for bipolar disorder is localized on chromosome 12q24. In order to obtain further support for this locus and to narrow the region of interest, distantly related patients with bipolar disorder from the Faroe Islands were investigated. The population of the Faroe Islands was founded by relatively few individuals, has been relatively isolated since its founding and may be a wellsuited population for genetic mapping of risk genes. Using a number of microsatelitte markers the study found evidence for allelic association, haplotype and chromosome segment sharing in the same region as previously suggested by haplotypes in two Danish families with bipolar disorder which showed significant linkage to chromosome 12q24.

Exon/intron boundaries, novel polymorphisms, and association analysis with schizophrenia of the human synaptic vesicle monoamine transporter (SVMT) gene

H Kunugi, S Ishida, A Akahane, S Nanko

The synaptic vesicular monoamine transporter (SVMT), alternatively vesicular monoamine transporter 2 (VMAT2), pumps cytosolic monoamines including dopamine, norepinephrine, serotonin, and histamine into synaptic vesicles. The SVMT protein plays a critical role in regulating neural transmission, and altered functions of SVMT may be related to the cause of several neuropsychiatric diseases. The authors determined exon/intron boundaries of the human SVMT gene and searched for sequence variations of the gene. They found two and four single nucleotide polymorphisms (SNPs) in exons and neighboring introns, respectively. The authors also examined whether novel polymorphisms are associated with the development of schizophrenia in a Japanese family-based association sample. However, they found no statistically significant association, although the sample size (50 families) is small and further investigations in a larger sample size are required. The current study suggests that there is no common polymorphism in the SVMT gene affecting the primary structure of the SVMT protein.

Voltage-gated calcium channel $\gamma 2$ subunit gene is not deleted in velo-cardio-facial syndrome JL Black, LE Krahn, SM Jalal

Velo-cardio-facial syndrome (VCFS) has been associated with schizophrenic symptoms in some patients and is caused by a deletion of part of chromosome 22. The voltage-gated calcium channel (VGCC) $\gamma 2$ subunit is also located on chromosome 22 and is near a possible marker for schizophrenia, which is called D22S278. The authors studied four healthy controls, four patients with VCFS, and one patient with both VCFS and schizophrenia to see if the $\gamma 2$ subunit gene was missing. All of the VCFS patients had the expected deletion on chromosome 22 but none showed a deletion of the $\gamma 2$ subunit. The location of the $\gamma 2$ subunit gene at the chromosome 22 position called 22q13.1 was confirmed. This study did not show a deletion of the $\gamma 2$ subunit gene as a distinguishing feature of their patient with VCFS and schizophrenia.

\section{Identification of a compound short tandem repeat stretch in the $5^{\prime}$-upstream region of the cholecystokinin gene, and its association with panic disorder but not with schizophrenia E Hattori, M Ebihara, K Yamada, H Ohba, H Shibuya, T Yoshikawa}

The cholecystokinin gene (CCK) is thought to play a role in the pathogenesis of panic disorder and schizophrenia. In this study, the authors have extended the 5 '-upstream sequence of the CCK gene, and identified a compound short tandem repeat (STR), which was polymorphic with ten different allele lengths. Dividing the STR alleles into three classes according to length, Long (L), Medium (M) and Short (S), produced strong genotypic (MM) and allelic (M) associations with panic disorder but not with schizophrenia. In the extended promoter region, the authors have detected the previously known $-36 \mathrm{C}>\mathrm{T}$ and $-188 \mathrm{~A}>\mathrm{G}$ single nucleotide polymorphisms (SNPs) and a new rare SNP, $-345 \mathrm{G}>\mathrm{C}$. Haplotypic distributions of the STR and SNPs -188 and -36 were significantly different between panic samples and controls, further suggesting that the novel STR or a nearby variant may confer susceptibility to panic disorder.

\section{Brain-derived-neurotrophic-factor (BDNF) stress response in rats bred for learned helplessness $B$ Vollmayr, $H$ Faust, $S$ Lewicka, FA Henn}

BDNF (brain-derived neurotrophic factor) has been implicated in stress-induced hippocampal adaptation and pathogenesis of depression. The authors used learned helplessness as an animal model of some features of depression. To enhance genetic predisposition they have inbred two Sprague-Dawley strains based on their behavior in learned helplessness testing: one 
which reacts with congenital helplessness to stress (cLH), and one which congenitally does not acquire helplessness (cNLH). These strains have lost their behavioral plasticity including their sensitivity to antidepressant treatment. The authors show here that in cLH rats acute stress does not induce a reduction of BDNF expression in the hippocampus which is observed in Sprague-Dawley and cNLH rats. All animals tested exhibited elevated corticosterone levels when stressed, an indication that in CLH rats regulation of BDNF expression in the hippocampal formation is uncoupled from corticosterone increase induced through stress. This may explain the lack of adaptive responses in this strain.

Treatment with interferon-alpha (IFN $\alpha$ ) of hepatitis C patients induces lower serum dipeptidyl peptidase IV activity, which is related to IFN $\alpha$-induced depressive and anxiety symptoms and immune activation

M Maes, S Bonaccorso, V Marino, A Puzella, M Pasquini, $M$ Biondi, $M$ Artini, C Almerighi, H Meltzer

The authors have shown that immunotherapy with cytokines, such as interleukin-2 (IL-2) or interferon- $\alpha$ $(\mathrm{IFN} \alpha)$ may induce depressive symptoms and depression in a considerable number of patients treated. Immunotherapy may also induce immune activation and may diminish the serum activity of dipeptidyl peptidase IV (DPP IV), an enzyme that catalyzes the cleavage of peptides which have behavioral effects and which modulate the activity of immune cells. In the present study the authors examined the effects of immunotherapy with IFN $\alpha$ on serum DPP IV activity in relation to induction of immune activation. The authors found that IFN $\alpha$-based immunotherapy significantly suppressed serum DPP IV 2-4 weeks and 1624 weeks after starting immunotherapy. The IFN $\alpha$ - induced suppression of serum DPP IV activity was sig357 nificantly correlated to IFN $\alpha$-induced increases in the MADRS and HAM-A and increases in the K/T quotient and serum IL-8. These results suggest that: (1) immunotherapy with IFN $\alpha$ suppresses serum DPP IV activity and induces immune activation and an increased catabolism of tryptophan; and (2) the changes in DPP IV are related to increases in severity of depression, anxiety and activation of the immune system.

Borna disease virus-specific circulating immune complexes, antigenemia, and free antibodies - the key marker triplet determining infection and prevailing in severe mood disorders L Bode, P Reckwald, WE Severus, $R$ Stoyloff, $R$ Ferszt, DE Dietrich, H Ludwig

An infectious cause of major affective disorders has been hypothesized for decades. A unique, evolutionary old RNA virus, Borna disease virus (BDV), is regarded as a candidate, due to analogous behavioral syndromes caused in different animal species. Human BDV infections have been demonstrated by serum antibodies, viral proteins and nucleic acid in white blood cells. However, their significance remained controversial based on low amounts and inconstant detection. The discovery of BDV-specific circulating immune complexes (CICs), corresponding with free plasma antigens (BDV -p40/-p24) and antibodies, provides new evidence for an important role of BDV in mood disorders. BDV-CICs indicate an infection rate of close to $100 \%$ in acutely depressed patients (major depression or bipolar disorder), vs up to $30 \%$ in the healthy population. Severity of depression correlates with persisting strength of CIC formation and antigenemia. Easy-to-use diagnostic tools have the potential to promote largescale clinical studies and epidemiology.

J Licinio, MD Editor 